\title{
DESENVOLVIMENTO NEUROPSICOMOTOR DE LACTENTES FILHOS DE MÃES QUE APRESENTARAM HIPERTENSÃO ARTERIAL NA GESTAÇÃO
}

\author{
Briana R. Dias" ${ }^{1}$ Ana Maria S.G. Piovesana2*, \\ Maria Augusta Montenegro², Marilisa M. Guerreiro ${ }^{3}$
}

\begin{abstract}
RESUMO - In trølução: A hipertensão gestacional é a maior causa de morte materna no país e pode cursar com encefalopatia hipóxico-isquêmica no concepto levando a subseqüentes manifestações neurológicas. Objetivo: Correlacionar a hipertensão gestacional com indicadores de risco para o desenvolvimento ne u ropsicomotor do lactente. Método: Foram avaliados 30 recém-nascidos, filhos de mães que apresentaram quadro de hipertensão gestacional, de forma consecutiva e prospectiva. Foram considerados como indicadores de risco os seguintes fatores: pequeno para a idade gestacional; sofrimento fetal agudo; início da hipertensão gestacional; recém-nascido a termo/pré-termo; índice de Apgar; presença de cianose central; necessidade de máscara de $\mathrm{O}_{2} ;$ cor do líquido amniótico. Este estudo cumpriu duas etapas. Na primeira, realizamos o exame neurológico entre as primeiras 48-72 horas de vida do neonato. Em uma segunda etapa, os pacientes foram submetidos a uma segunda avaliação neuroclínica entre os 7 e 15 meses de vida. Resultados: Seis recém-nascidos avaliados apresentaram alterações ao exame neurológico nas primeiras 72 horas de vida. Dos indicadores analisados, apenas o índice de Apgar de risco se correlacionou com o exame neu rológico neonatal alterado de forma estatisticamente significativa. Os demais indicadores não apresentaram correlação positiva. Todas as crianças reavaliadas na segunda etapa demonstraram exame neurológico e desenvolvimento neuropsicomotor normais, não sendo possível qualquer correlação com os indicadores de risco. Conclusão: Os nossos dados sugerem que a hipertensão gestacional per se parece não ser suficiente para causar danos neurológicos importantes ao concepto.
\end{abstract}

PALAVRAS-CHAVE: hipertensão gestacional, desenvolvimento neuropsicomotor, índice de Apgar, sofrimento fetal agudo, encefalopatia hipóxico-isquêmica.

\begin{abstract}
Neuropsychomotor development of infants born of mothers with gestational hypertension
ABSTRACT - Introduction Gestational hypertension is a major cause of maternal death in our country and may be associated with neonatal hypoxic-ischemic encephalopathy with serious neurological complications. Objective: To correlate gestational hypertension with risk factors of neuropsychomotor development in infants. Method: This was a prospective study. We evaluated 30 consecutive infants born of mothers with gestational hypertension. The following risk factors were considered: small for gestational age; fetal asphyxia; age of onset of gestational hypertension; term/p rete rmnewborn; Apgar scores; central cyanosis; $\mathrm{O}_{2}$ mask; meconium. The study followed two steps. In the first step, newborns underwent neurological examination soon after birth (48-72 hours of life). In the second step, children underwent another ne u rological assessment between 7 and 15 months of life. Results: Six newborns presented neurological signs on the first evaluation. The only risk factor that showed a significant correlation with the neurologic examination was the Apgar score. Other risk factors did not show any correlation. All children evaluated on the second step of the study showed normal neurological development and examination, which did not allow any correlation with risk factors. Conclusion: Our data suggest that gestational hypertension per se is not sufficient to cause fetal neurological impairment.
\end{abstract}

KEY WORDS: gestational hypertension, neuropsychomotor development, Apgar score, fetal asphyxia, hypoxic-ischemic encephalopathy.

Departamento de Neurologia, Faculdade de Ciências Médicas Universidade Estadual de Campinas, Campinas SP, Brasil (FCMUNICAMP): ${ }^{1}$ Aluna de Medicina; ${ }^{2}$ Professor Doutor; ${ }^{3}$ Professor Associado; ${ }^{*}$ in memorian. Apoio: CNPq.

Recebido 2 Agosto 2004, recebido na forma final 20 Dezembro 2004. Aceito 10 Março 2005.

Dra. Marilisa M. Guerre iro - Departamento de Neurologia, FCM-UNICAMP / Caixa Postal 6111 - 13083-970 Campinas SP - Brasil. E-mail: $m m g @ f c m . u n i c a m p . b r$ 
A hipertensão gestacional ou doença hipertensiva específica da gestação caracteriza-se pelo aumento da resistência vascular periférica acarre tando aumento nos níveis tensionais da pressão art erial para valores em torno de $140 \mathrm{mmHg}$ (sístole) e $90 \mathrm{mmHg}$ (diástole) 1 . Entre as principais complicações maternas, podemos encontrar edemas e proteinúria. A hipertensão na gestação é a maior causa de morte materna no país, sendo responsável por $35 \%$ delas. Em relação à mortalidade perinatal, a taxa nacional é 150/1000 partos. Em recente trabalho epidemiológico sobre a relação entre a préeclâmpsia e encefalopatia neonatal constatou-se que das grávidas que apresentaram quadro de préeclâmpsia, 14,5\% dos neonatos apresentaram encefalopatia ${ }^{2}$.

A encefalopatia hipóxico-isquêmica é uma encefalopatia não prog ressiva conseqüente à síndrome hipóxica-isquêmica, causada por uma mistura da redução de oxigenação de sangue com um aumento de dióxido de carbono (asfixia) e falta de perfusão nos tecidos (isquemia) ${ }^{3}$.

Outras possíveis repercussões neurológicas podem variar de acordo com a idade gestacional ${ }^{4}$. No recém-nascido a termo, a isquemia se projeta com lesões sobre a massa cinzenta principalmente sob reo córtex cerebral. A distribuição da necrose não ocorre de forma uniforme, afetando preferencialmente o hipocampo. No recém-nascido pré-termo, a hipóxia pode levar à leucomalácia periventricular. Clinicamente corresponde com freqüência aos quadros de paralisia cerebral do tipo diplegia espástica ${ }^{5-7}$.

O objetivo do presente estudo é correlacionar a hipertensão gestacional com indicadores de risco para o desenvolvimento neuropsicomotor do lactente.

\section{MÉTODO}

Foram avaliados de forma consecutiva e prospectiva. 30 recém-nascidos, filhos de mães que apresentaram quadrode hipertensão gestacional. Foram excluídas da amostra: gestantes que apresentaram cardiopatias associadas ao aumento pressórico; associação de co-morbidades como diabetes, tabagismo, etilismo, dependência química e patologias renais. Foram também excluídos os neonatos com: síndromes genéticas; malformações congênitas; malformações do sistema nervoso central (SNC); e lesões do sistema nervoso periférico.

Foram considerados como indicadores de risco os seguintes fatores: pequeno para a idade gestacional; sofrimento fetal agudo; início precoce da hipertensão gestacional (<28 semanas); prematuridade; índice de Apgar (sendo considerado Apgar de risco quando $<6$ no $5^{\circ} \mathrm{mi}-$ nuto); presença de cianose central; necessidade de máscara de $\mathrm{O}_{2}$; cor do líquido amniótico.

Sofrimento fetal agudo foi definido como: presença de mecônio + anormalidade dos batimentos cardíacos fetais + distúrbio do equilíbrio ácido-básico ${ }^{11}$.

Encefalopatia hipóxico-isquêmica foi definida como: evidência de sofrimento fetal agudo + depressão ao nascimento + persistência dessas alterações nas primeiras horas ou dias de vida + distúrbio ventilatório + resposta pupilar intacta + resposta oculomotora intacta + hipotonia + crises $^{12}$.

Este estudo cumpriu duas etapas. Na primeira, realizamos o exame neurológico entre as primeiras $48-72$ horas de vida do neonato. O roteiro seguido foi o de exame neurológico do recém-nascido a termo ${ }^{5}$ e exame neurológico do recém-nascido prematu ro $^{8}$. Esta avaliação foi realizada no CAISM-Unicamp nos setores do alojamento conjunto, patologia obstétrica, e UTI neonatal intensivo e semi-intensivo.

Em uma segunda etapa, os pacientes foram submetidos a uma segunda avaliação neuroclínica entre os 7 e 15 meses de vida. Nesta etapa, o roteiroseguido foi o exame neurológico do lactente ${ }^{9}$ associado à aplicação do teste de Denver ${ }^{10}$. As consultas foram realizadas no próprio ambulatório de neurologia do Hospital de Clínicas da Unicamp.

O período de inclusão para a primeira etapa foi de outubrode 2002 a junho de 2003 e a reavaliação neuroclínica ocorreu durante os meses de janeiro a março de 2004.

\section{RESULTADOS}

Seguimos 30 mães que apresentaram quadro clínico de hipertensão gestacional. O aumento da pressão arterial precoce, abaixo de 28 semanas, esteve presente em 11(36\%) gestantes. A situação de primigestas correspondeu a $37 \%$ das mães analisadas e $43 \%$ tinham idade considerada tardia, ou seja, maiores de 32 anos. O número de gestantes que já apresentava quadro hipertensivo antes da gestação correspondeu a $27 \%$ e, sendo assim, $73 \%$ apresentaram quadro de pré-eclâmpsia.

Foram avaliados 30 neonatos entre 48-72 horas de vida. A prematuridade ocorreu em nove (30\%) deles e 21 (70\%) foram recém-nascidos a termo. Incluímos cinco (17\%) neonatos considerados pequenos para a idade gestacional. O sofrimento fetal agudo foi identificado em 10 (33\%) recém-nascidos sendo que o Apgar considerado de risco, abaixo de seis no $5^{\circ}$ minuto, foi constatado em sete dos 30. A cianose central ao nascimento foi identificada em 20 (67\%) neonatos sendo que em 25 $(83 \%)$ deles foi necessária a aspiração de vias aé- 
reas superiores. Máscara aberta de $\mathrm{O}_{2}$ foi utilizada em 20 recém-nascidos. Não foi identificada qualquer anormalidade quanto ao perímetro craniano.

Quanto ao exame neurológico, as alterações eram consideradas quando o neonato apresentava distúrbios e assimetria da motricidade voluntária e estimulada e/ou reflexos de liberação piramidal e/ou hipotonia. Este último foi adequado para dife renciar a hipotonia devido à prematuridade $\mathrm{e}$ ao choque do nascimento. Portanto, 6/30 (20\%) apresentaram alterações do exame neurológico tendo prevalência a hipotonia, 6/6. Os reflexos de liberação piramidal estiveram presentes em 2/30 $(6 \%)$ e um neonato apresentou o quadro de encefalopatia hipóxico-isquêmica. A Tabela 1 expõe as alterações encontradas no exame neurológico de seis neonatos. Assim, 20\% dos recém-nascidos ava- liados apresentaram alterações ao exame neurológico nas primeiras 72 horas de vida.

Analisamos vários fatores de risco no período neonatal e comparamos os dados entre os recémnascidos com exame neurológico normal ou alterado. As informações constam da Tabela 2. Entre os fatores analisados, o índice de Apgar foi o único que mostrou correlação positiva com o exame neurológico alterado, uma vez que neonatos com Apgar de risco tiveram chance significativamente maior de apresentarem alguma manifestação neurológica.

Na segunda fase do projeto, foram convocados os mesmos 30 pacientes da primeira fase, tendo comparedido apenas 15 (50\%) deles. Dos seis neonatos que apresentaram manifestações neurológicas, quatro deles compareceram para serem rea-

Tabela 1. Manifestações neurológicas anormais em seis neonatos.

\begin{tabular}{lll}
\hline $\begin{array}{l}\text { Número do } \\
\text { paciente }\end{array}$ & $\begin{array}{l}\text { Manifestações neurológicas no } \\
\text { período neonatal }\end{array}$ & $\begin{array}{l}\text { Exame neurológico e teste de } \\
\text { Denver entre 7 e 15 meses }\end{array}$ \\
\hline 2 & $\begin{array}{l}\text { Hipotonia global } \\
\text { Hiperreflexia }\end{array}$ & Normais \\
7 & $\begin{array}{l}\text { Hipotonia axial } \\
\text { Hipertonia membros } \\
\text { Crise epiléptica tônica e apnéia }\end{array}$ & Normais \\
11 & Hipotonia global & Normais \\
12 & Hipotonia global & Normais \\
19 & Hipotonia global & Normais \\
& Irritabilidade & \\
24 & Paresia membro superior direito & Nipotonia maior à direita
\end{tabular}

Tabela 2. Análise de fatores de risco no período neonatal.

\begin{tabular}{lccc}
\hline Fatores de risco & EN normal & EN alterado & $p$ \\
\hline PIG/Não PIG & $4 / 20$ & $1 / 5$ & 1,000 \\
SFA (N/S) & $18 / 6$ & $2 / 4$ & 0,052 \\
Início PA (P/T) & $9 / 15$ & $2 / 4$ & 0,849 \\
RNT/RNPT & $18 / 6$ & $3 / 3$ & 0,231 \\
Apgar (nl/risco) & $21 / 3$ & $2 / 4$ & 0,0050 \\
Cianose central (N/S) & $10 / 14$ & $0 / 6$ & 0,052 \\
Máscara de O2 (N/S) & $10 / 14$ & $0 / 6$ & 0,052 \\
LA (N/Alt) & $22 / 2$ & $4 / 2$ & 0,107 \\
\hline
\end{tabular}

EN, exame neurológico; PIG, pequeno para a idade gestacional: SFA, sofrimento fetal agudo; N, não; S, sim; $\mathrm{P}$, precoce; T, tardio; RNT, recém-nascido a termo; RNPT, recémnascido pré termo; NL, normal; LA, líquido amniótico; Alt, alterado. 
valiados. A idade variou entre sete e 15 meses sendo corrigidas para os testes no caso de pre maturidade. Na avaliação da linguagem, todos se a $p$ resentaram adequados para a faixa etária dent ro do limite da normalidade, variando de lalação às primeiras palavras. Dez de 15 pacientes $(67 \%)$ permaneciam em pé com apoio e assim como os demais estavam adequados quanto à postura e equilíbrio. Na avaliação da locomoção, apenas dois (13\%) já estavam andando. A preensão voluntária apresentou-se normal para todos os lactentes e variou de dígito palmar à preensão em pinça superior. O tono muscular também se apresentou n o rmal considerando também os casos de hipotonia fisiológica. As atividades reflexas primitivas e os reflexos miotáticos estavam dentro dos limites da normalidade. Não foi observada qualquer alteração quanto ao perímetro craniano. $O$ teste de Denver revelou que as provas dos setores pessoalsocial, motor adaptativo, linguagem e motor foram consideradas normais para todos os lactentes avaliados. Em outras palavras, todos os 15 lactentes re-examinados apresentaram o desenvolvimento neuropsicomotor normal e não foi possível a correlação com os indicadores de risco nesta segunda etapa.

\section{DISCUSSÃO}

Sofrimento fetal agudo ocorreu em $33 \%$ de nossa casuística. Isto está de acordo com o fato de que mães hipertensas apresentam grande chance de terem filhos com sofrimento fetal ${ }^{13,14}$. Entretanto, apenas um neonato recebeu o diagnóstico de encefalopatia hipóxico-isquêmica. Este achado corrobora a informação de que a maioria dos recém-nascidos que apresenta insulto hipóxico-isquêmico intra-uterino não exibirá subseqüente evidência neurológica de dano cerebral ${ }^{12}$. A criança que preencheu os critérios para encefalopatia hipóxico-isquêmica foi re-examinada na segunda etapa do presente trabalho e seu exame neurológico revelou-se normal. Outros autores também já observaram que a maioria dos neonatos que recebem o diagnóstico de encefalopatia hipóxico-isquêmica acaba tendo boa evolução neurológ ica ${ }^{12,15}$. Assim, os nossos dados reforçam a observação de que existe um grande funil em termos de gravidade: a hip e rtensão gestacional causa sofrimento fetal agudo em uma porcentagem de neonatos; a maioria desses neonatos nada terá em sua evolução neurológica; entretanto, em alguns casos o sofrimento fetal agudo levará a encefalopatia hipóxico-isquê- mica; a maioria destes felizmente também não a p resentará alteração neuropsicomotora e, assim, apenas um número reduzido de pacientes acabará tendo manifestações neurológicas no desenvolvimento.

Esse mesmo raciocínio pode ser atingido observando-se a evolução das manifestações neurológicas anormais presentes ao nascimento em seis de nossos pacientes. Todos tiveram exame neurológico normal no segundo exame, o que reforça o ponto de que apenas uma minoria de crianças cursará com complicações neurológicas decorrentes da hipertensão gestacional.

Nenhuma criança que compôs a nossa casuística teve sangramento no SNC ou complicações neonatais graves como depressão cardíaca, respiratória ou de consciência. Isto se deve provavelmente à pequena casuística desse estudo. De qualquer forma, a ausência de complicações perinatais graves levou-nos a aventar a hipótese de que quando a hipertensão gestacional não cursa com complicações mais sérias, os neonatos apresentam bom desenvolvimento neurológico. Em outras palavras, a hipertensão gestacional per se parece não ser suficiente para causar danos neurológicos importantes ao concepto. Por outro lado, crianças com sofrimento fetal agudo que não desenvolvem paralisia cerebral podem ter risco aumentado de desenvolver quadros neurológicos sutis, tais como dificuldade no aprendizado ${ }^{16}$.

Dos fatores de risco analisados o único que se co rrelacionou com o exame neurológico ao nascimento foi o índice de Apgar. Neonatos com Apgar de risco tiveram maior chance de apresentar alterações ao exame neurológico. Apesar de alguns autores apontarem este índice como um bom fator preditivo do prognóstico ${ }^{16-18}$, a maioria acredita que há uma tênue relação entre asfixia fetal e baixos índices de Apgar ${ }^{19-22}$. O nosso achado de que o índice de Apgar foi o único fator de risco que of e receu correlação com o exame neonatal deve, portanto, ser visto com cautela.

Todos os lactentes avaliados na segunda etapa apresentavam exame neurológico e desenvolvimento neuropsicomotor normais. Isto não nos permitiu qualquer correlação com os indicadores de risco analisados. Como já comentado, mesmo os neonatos que tiveram alguma alteração neurológica ao nascimento acabaram se desenvolvendo adequadamente. 


\section{Concluímos que a hipertensão gestacional não costuma afetar o concepto a não ser que os níveis pressóricos elevados sejam suficientes para pro- vocar danos tais como insultos vasculares.}

\section{REFERÊNCIAS}

1. Resende J. Doença hipertensiva específica da gestação: pré-eclâmpsia e eclâmpsia. Obstetrícia 1995;22:598-642.

2. Impey L, Greenwood C, Sheil O, MacQuillan K, Reynolds M, Redman C. The relation between pre-eclampsia at term and neonatal encephalopahty. Arch Dis Child 2001;85:170-172.

3. Rufo MC, Palencia RL. Encefalopatia hipóxico-isquémica del recién nacido a término: recientes avances, marcadores de hipoxia y opciones terapéuticas. Rev Neurol 2000;31:617-623.

4. Marques-Dias MJ. Encefalopatias hipóxico-isquêmicas e hemorragias intracranianas. In Diament A, Cypel S (eds). Neurologia infantil. São Paulo: Livraria Atheneu, 1999:667-674.

5. Diament A. Exame neurológico do recém-nascido de termo. In Diament A, Cypel S (eds). Neurologia infantil. São Paulo: LivrariaAtheneu, 1999:10-21.

6. Volpe JJ. Hipoxic-isquemic encephalopathy: neuropathology and pathogenesis. In Volpe JJ (ed). Neurology of the newborn, $4^{\text {th }}$ edition. Philadelphia: W.B. Saunders, 2001:296-330.

7. Keeling JW. Acquired diseases of the nervous system. Fetal Neonatal Pathology 1993;24:571-593.

8. Gherpelli JL. Avaliação neurológica do recém-nascido prematuro. In Diament A, Cypel S (eds). Neurologia infantil. São Paulo: Livraria Atheneu, 1999:22-32.

9. Diament A. Exame neurológico do lactente. In: Diament A, Cypel S (Eds). Neurologia infantil. São Paulo: Livraria Atheneu, 1999:33-61.

10. Frankenburg WK. Denver developmental screening. J Ped 1967;71: 181-191.
11. Volpe JJ. Hypoxic-ischemic encephalopathy: intrauterine assessment. In: Volpe JJ (Ed). Neurology of the newborn. $4^{\text {th }}$ edition. Philadelphia: W.B. Saunders Company, 2001:277-295.

12. Volpe JJ. Hypoxic-ischemic encephalopathy: clinical aspects. In Volpe JJ (ed). Neurology of the newborn, 4th edition. Philadelphia: W.B. Saunders, 2001:331-394.

13. Mutch LM, Moar VA, Ounsted MK, Redman CW. Hypertension during pregnancy, with and without specific hypotensive treatment: I. Perinatal factors and neonatal morbidity. Early Hum Dev 1977;1:47-57.

14. Spinillo A, Stronati M, Ometto A, Fazzi E, Lanzi G, Guaschino S. Infant neurodevelopmental outcome in pregnancies complicated by gestational hypertension and intra-uterine growth retardation. J Perinat Med 1993;21:195-203.

15. Gonzalez de Dios J, Moya M. Perinatal asphyxia, hypoxic-ischemic encephalopahty and neurological sequelae in full-term newborns: II. Description and interrelation. Rev Neurol 1996;24:969-976.

16. Moster D, Lie RT, Markestad T. Joint association of Apgar socres and early neuonatal symptoms with minor disabilities at school age. Arch Dis Child Fetal Neonatal 2002;86:16-21.

17. Gross SJ, Kosmetatos N, Grimes CT, Williams ML. Newborn head size and neurological status: predictors of growth and development of low birth weight infants. Am J Dis Child 1978;132:753-756.

18. Skouteli HN, Dubowitz LM, Levene MI, Miller G. Predictors for survival and normal neurodevelopmental outcome of infants weighing less than 1001 grams at birth. Dev Med Child Neurol 1985;27:588-595.

19. Ruth VJ, Raivio KO. Perinatal brain damage: predictive value of metabolic acidosis and the Apgar score. BMJ 1988;297:24-27.

20. Marlow N. Do we need an Apgar score? A rch Dis Child 1992;67: 765-767.

21. Silverman F, Suidan J, Wasserman J, et al. The Apgar score: is it enough? Obstet Gynecol 1985;66:331-336.

22. Marrin M, Paes BA. Birth asphyxia: does the Apgar score have diagnostic value? Obstet Gynecol 1988;72:120-123. 